\title{
Mathematical Tutorial System (MTS) Using Fuzzy Logic and Multi-Agent System
}

\author{
Gandhimathi. K, \\ Assistant Professor / CSE, \\ Idhaya Engineering College for Women, \\ Chinnasalem - 606 201, Villupuram District, \\ Tamilnadu, India \\ Sr. J. Arockia Jaya \\ Assistant Professor / CSE, \\ Idhaya Engineering College for Women, \\ Chinnasalem - 606 201, Villupuram District, \\ Tamilnadu, India
}

\begin{abstract}
Appropriate use of computer technology in the learning process has been lacking and many web-based and standalone tutorial systems in today's market-place do not provide material that teaches students in an enjoyable and effective way. The Mathematical Tutoring System (MTS) is one which provides direct customized feedback to students. It also guides and monitors student progress. Our aim is to design an instructional planner for an intelligent tutoring system. We use the combined approach of Fuzzy logic and Multiagent System (MAS). The fuzzy logic determines the next level (objective) to be presented to the student based on the current and past performance of the objectives. The Multiagent system on the other hand decides the set of activities to be presented in the objective. The educational objective of the MTS presented in our work is the number concept and the objectives of Problems in primary education curriculum.
\end{abstract}

Keywords: Fuzzy Logic, Multi Agent System, Mathematical Tutorial System, Similarity Calculation

\section{INTRODUCTION}

The educational objective of the MTS presented in our work is the number concept and the objectives of Problems in primary education curriculum. The student knowledge varies from one person to another. So the Instructional Planner for an MTS must dynamically adapt to each students to teach the number and addition concepts. So we used the combined approach of fuzzy logic and multiagent systems.

Each student will be given a username and password after the registration process. The set of activities

\author{
Yogarani. A \\ Assistant Professor / EEE, \\ Idhaya Engineering College for Women, \\ Chinnasalem - 606 201, Villupuram District, \\ Tamilnadu, India
}

\author{
Ponmathi. M \\ Assistant Professor / CSE, \\ Idhaya Engineering College for Women, \\ Chinnasalem - 606 201, Villupuram District, \\ Tamilnadu, India
}

carried out by the student varies depending upon their knowledge. Basically the students are categorized into kindergarten and Primary. The primary is in turn classified as:

- Fear of Failure

- Motivated

- Hyperactive

Each student will be among one of the above mentioned classifications. The classification is done based on the pre-test. Then a set of objectives are selected for a particular student. Each objective consists of set of activities which are to be carried out by the student during the path of learning. Based on the performance in an objective the student will be guided to the respective levels.

The fuzzy logic determines the next level (objective) to be presented to the student based on the current and past performance of the objectives. The work of multiagent system is to select the appropriate activity to be presented to the student for that objective. This performance of each student is monitored by the MTS and it is updated in the Student Database. The system uses multimedia such as video and images which helps the student in acquiring the concepts.

The student can quit at any time. On quitting, the system will record the student's progress. When he enters next time, he will be guided according to his previous stored information.

Fuzzy is to solve a new problem by recalling a previous situation which is similar and by reusing the information and knowledge of that situation. It will attempt to find the case or cases in the case base that most closely match the current query case [1].

From the case base previous identical case is retrieved. If the current problem and previous problem are 
identical then the solution of the past problem will be returned as the current problem's solution. If the retrieved case is not identical to the current case the differences between the current case and the retrieved case must be identified and then the solution associated with the retrieved case is modified taking into account these differences. The solution returned for the current problem will be tried in the appropriate domain setting.

\section{RELATED WORK}

Weizenbaum's (1966) [3] designed ELIZA which tried to get the patient to do the talking by asking the patient questions about the patient's verbal contributions to the exchange. Conversation partners are expected to answer questions, so a therapist's question readily turns the spotlight onto the patient. ELIZA turned the patient's assertions into a therapist's questions by simple syntactic transformational rules. That is, ELIZA detected keywords and word combinations that triggered rules, which in turn generated ELIZA'S responses.

Arthur C.Graessar et al (2004) [3] discussed the learning environment that tutors students by holding a conversation in natural language. Auto Tutor has been developed for Newtonian qualitative physics and computer literacy. MTS design was inspired by explanation-based constructivist theories of learning, intelligent tutoring systems that adaptively respond to student knowledge, and empirical research on dialogue patterns in tutorial discourse. Auto Tutor presents challenging problems (formulated as questions) from a curriculum script and then engages in mixed initiative dialogue that guides the student in building an answer.

K.J.Satao et al (2010) [4] designed a tutorial to enable the students learning the language via computerbased tutorials to communicate with the tutor without any need to be fluent in each other's language. This model enables an Indian user to generate computer-based tutorials without any programming knowledge, serves as a multiple domain tutor to English monolingual users who are learning English, and the prototype also incorporates English-Hindi translation ability. Based on reviews of previous work, such features are usually incorporated separately in different systems and applications.

Meeta Dewangan et al (2011) [6] designed a English tutor helps the learner understand and practice basic conversation skills with vocabulary, how to greet people, knowing more about them, how to make use of the language in their daily routine, how to invite people and how to request or ask for a favour from them. Making the user aware of the scenarios which the user come across when going around a place, about basic necessities, making him know the objects he may come across, things he may need while planning a journey, skills of describing a place, people the user have across etc, about the way of answering a telephone call, basic eti-quettes of talking on phone, extempore questions and their model answers.

\section{ARCHITECTURE OF MTS}

The proposed Instructional Planner (IP) for an Mathematical Tutoring System consist of four modules

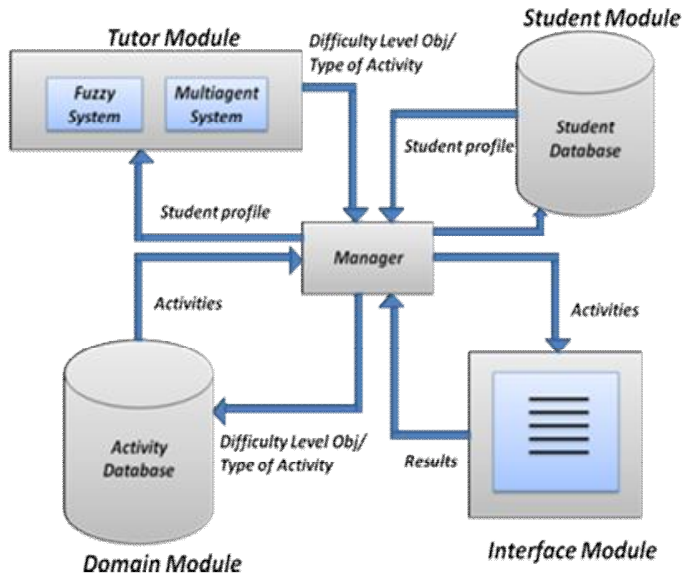

Fig 1 Architecture of an MTS

\subsection{Interface Module}

The interface module helps the student to interact with the MTS, usually through a graphical user interface which helps the students for learning and taking the test.

\subsection{Student Module}

This module monitors the student's performance. The module basically is a database. It contains all the data and information regarding the student. These data are used to choose both the appropriate subsequent subject and the educational methodology or strategies. Basically it can be divided two fundamental components, such as the profile and the history.

- The profile consists of student personal information such as Name, Password, and Category etc...

- The history covers those variables related to previous concepts that the student knows, as well as the progress exhibited (repeated exercises, omitted tasks, rate of progress between activities, learning style, etc...)

\subsection{Tutor Module}

This module identifies the new problem. The module monitors the performance of the students to find the new problem. This problem will be given as a case into the activity database.

\subsection{Domain Module}

This module consists of the activity database. Activity database consists of various cases with the solution from which the most relevant case will be retrieved to find the new solution by using fuzzy logic. This solution can be used to choose the relevant materials for the students based on the student knowledge and current performance.

It also consists of the Activity Database. So, it can be called as Expert Module. This module contains the knowledge about the area of study. It contains the specific and detailed knowledge of the application domain as obtained from human experts. Every concept related to the learning objectives is described. In Mathematics, the mechanisms for learning concepts are specified.

Therefore, for each student several tasks are specified. These tasks are chosen based on the learning objectives for this student. 


\section{MATHEMATICAL TUTORIAL SYSTEM}

In this section we are going to discuss about the phases used in the MTS which helps the users to learn Mathematics. In this system there are two phases namely, learning phase and testing phase [6]:

\subsection{Learning Phase}

In this phase material will be provided to the student for learning. If the students were in the Kinder Garden then the material which can teach the numbers will be provided to them. If the students were in the Primary school then the material which can teach addition, subtraction, multiplication, division etc., will be provided to them for learning in an efficient way.

The main purpose of this phase is to familiarize the student with new material and to absorb the mathematical problems that your tutor considers most relevant to the topic.

\subsection{Testing Phase}

Test is used to evaluate student performance. The test will be given from the material from which they have learned. We are not interested in testing how well the students remember rather we are interested in testing how well the students actually use the Mathematics in real world entities.

\section{ROLE OF FUZZY LOGIC IN MTS}

The Fuzzy Instructional Planner consists of a set of fuzzy systems that will infer the sequence of objectives to be performed by the student. This sequence is based on the results obtained by the student. Since the teaching of the number, addition concepts entails covering a series of objectives, as many fuzzy systems must be built.

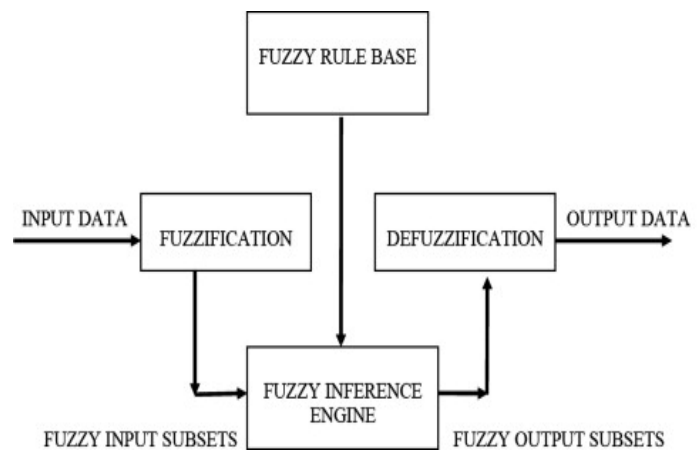

Figure 2: Fuzzy System with input and output

\section{Inputs and Outputs of Fuzzy System Input:}

The two inputs to the fuzzy system are

1. Activity Success Rate (ASR) -percentage of right answers provided by a student.ASR is a specific input whose value is between 0 and 1 (for example, if the student correctly answers $75 \%$ of the activities, this input will have a value of 0.75 )
2. ST- a variable to indicate the student's progress (i.e. ASR of previous objective)

Output:

Next Objective to be presented to the student

\subsection{Fuzzification}

Converting the crisp values into the corresponding fuzzy input (VeryLow, Low, Average, High, VeryHigh)

$\mathrm{ASR}=$ Number of questions answered correctly

Total Number of questions

\subsection{Fuzzy Proposition}

Terms like ASR is Low, ASR is High and ST is Advance are called as fuzzy proposition.

\subsection{Rule Base}

A convenient way of representing the set of rules is by using a table, where each square represents the linguistic value of the consequent of a rule, and the column to the left and the top row contain the linguistic values of the variables of the antecedent.

Table 1: Rule base for a student with a fear of failure

\begin{tabular}{llllll}
\hline ASR \ST & Very Low & Low & Average & High & Very High \\
Go back & Go back & Go back & Go back & Stay & Stay \\
Stay & Go back & Go back & Stay & Stay & Advance \\
Advance & Go back & Stay & Stay & Advance & Advance \\
\hline
\end{tabular}

Table 2: Rule base for a motivated student

\begin{tabular}{llllll}
\hline ASR \ST & Very Low & Low & Average & High & Very High \\
Go back & Go back & Go back & Stay & Stay & Stay \\
Stay & Go back & Stay & Stay & Stay & Advance \\
Advance & Stay & Stay & Stay & Advance & Advance \\
\hline
\end{tabular}

Table 3: Rule base for a hyperactive student

\begin{tabular}{llllll}
\hline ASR ST & Very Low & Low & Average & High & Very High \\
Go back & Go back & Go back & Stay & Stay & Advance \\
Stay & Go back & Stay & Stay & Advance & Advance \\
Advance & Stay & Stay & Advance & Advance & Advance \\
\hline
\end{tabular}

\subsection{Fuzzy Inferencing}

Combines the facts obtained from the fuzzification with the rule base and conducts the fuzzy reasoning process.

\subsection{Linguistic Descriptions}

The linguistic terms ASR and ST have the following values

ASR - VeryLow,Low,Average,High,Very High

ST- Go Back,Stay,Advance

\subsection{Working of Fuzzy Logic}

Once the set of activities is presented by the Multiagent System, the student will perform those activities 
and the values are fuzzified (ASR).ST value is obtained from the previous objective. Based on the values the Fuzzy Inferencing is done and the output is given to the aggregator. The aggregator receives input from two fuzzy systems and calculates the next level of objective to be presented to the student. Fuzzy System with aggregator is shown in fig 3.

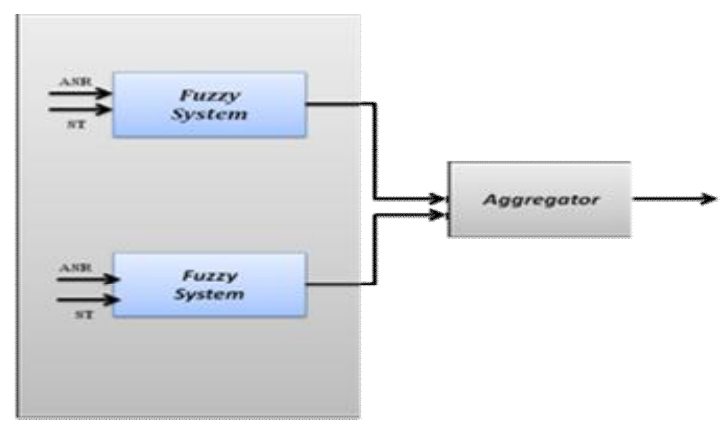

Figure 3 Fuzzy System with aggregator

The aggregator receives input from the fuzzy system. This step takes place before defuzzification. The aggregator work is to derive a conclusion form the consequent part of fuzzy rules. The aggregator is the output of the fuzzy system to take a decision. The functions used are Min, Max and average.

\section{MULTIAGENT SYSTEMS}

A MultiAgent System (MAS) is a system in which coexist a set of agents that interact with each other. Each agent has MTS own objectives and must cooperate with or compete against other agents in order to carry out these objectives.Since in a MultiAgent System no agent has direct control over other agents, a method for coordinating agents and resolving conflicts is necessary. This implies the introduction of a negotiation concept, which can be defined as a process by means of which a common decision is made by two or more parties. The parties involved first enunciate their contradictory demands and eventually move towards an agreement by means of a process of concessions or by searching for new alternatives. Different negotiation mechanisms exist. Different negotiation mechanisms exist. The instructional planner presented in this paper uses an auction-based mechanism, which offers a greater speed in attaining a solution.

Once the Objective is determined by the fuzzy logic the next task is to choose the type of activity within that objective that is going to be presented next to the student. Each objective consists of three types of activities. In our project there are three activity agents in which each agent has MTS own set of activities. Each activity agent computes the following function:

Function_Act $=\mathrm{p} 1 * \mathrm{Num}_{\mathrm{i}} \mathrm{Actv}_{\mathrm{i}}+\mathrm{p} 2 *$ Degree_Ful_Act $_{\mathrm{i}}$ Where

Num_Actv $\mathrm{i}_{\mathrm{i}}$ : number of activities done of type $\mathrm{i}$ versus the number of activities done for that objective.

Degree_Ful_Act $t_{\mathrm{i}}$ : percentage of right answers by the student for the type of activity $i$.

p1, p2: weight coefficients, whereP1 $=0.7, \mathrm{P} 2=0.3$

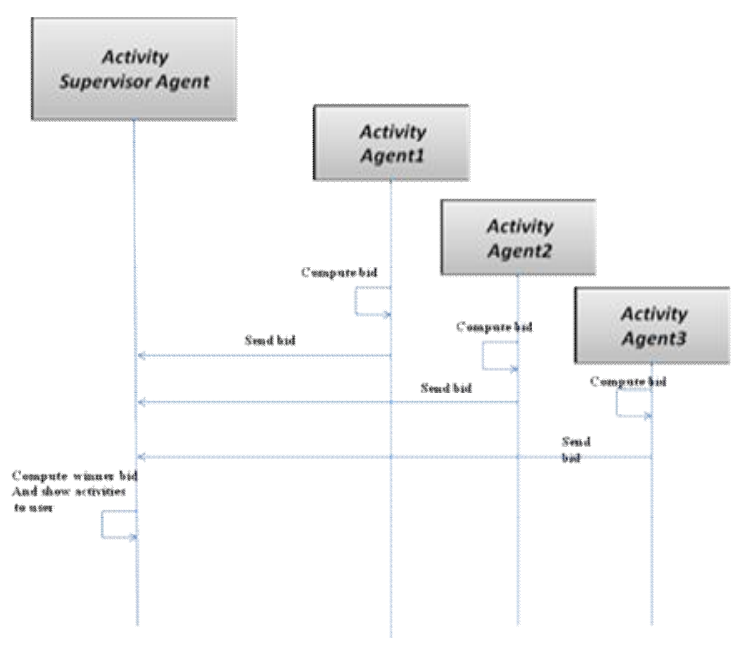

Figure 4 Activity Agent Sequence

As shown in the above figure 4 each activity agent will send the bid value to the Activity Supervisor Agent. The Activity Supervisor Agent selects two activities for an objective using the Open-Cry Descending Price Auction which chooses the lowest bid values. The chosen activities are displayed to the user.

Initially all the three agents have the same bid value as 0 because the student has not yet performed any activities. In such conditions the agent must choose two activities to be presented to the student. The domain module consist of number of tables in which each table consist of set of 30 activities (questions). The agent one is responsible for first 10 activities. The agent two is responsible for next 10 questions. The agent three is responsible for the next 10 questions. The questions in the table are arranged in a priority order in which first 10 questions have higher priority next 10 have lower priority then the first similarly for the next 10 questions. So initially the agent 1 and agent 2 are selected and their activities are given to the student. The performance of the student for each activity is recorded. When the student gets the same objective next time the student is presented with activities in which he haven't performed well.

\section{RESULT}

The qualitative validation relied on a series of cases (observations) for both the fuzzy and MultiAgent Systems that allowed for the results obtained from the tutorial simulation to be compared with what the experts would propose for said cases. The series of cases complied with the two essential requirements for the sample to be significant: quantity (that the number of cases be significant) and representativeness (that the cases exhibit variety within the application domain). These cases were validated by a group of experts, which offered the advantage of having the opinions of several experts (as opposed to that of a single expert or a consensus opinion). This results in fewer errors and also allows for the degree of agreement or similarity between the experts' responses to be compared. One sample case for validating the fuzzy system is as follows: consider a hyperactive student doing 
medium-level activities with a bad record of progressing through said activities. If, when doing new activities, his correct answer percentage is $50 \%$, what should be the next difficulty level assigned to the student? Should he be kept at the same level, dropped to the low level or raised to the high level? The statistical measures used for the quantitative validation are measures of agreement that compare the results of the tutorial with each expert's interpretation for the cases proposed. Fig. 5 shows a summary of the validation results for the fuzzy system that is used to determine the level of the next activity to be shown to the student.

The percentage of right answers given by the students for these activities is shown in Fig. 6. Every concept resulted in percentages above $60 \%$, though the ordering activities posed the greatest challenge to the students. The students' understanding of the action to be performed in the MTS was also evaluated for almost every activity presented, revealing more doubts regarding the mathematical than the technological concepts (Fig. 6). Also analyzed was the students' concentration when working with the MTS, since the interest and attention shown by the students when doing the activities has an obvious bearing on the correctness of their answers. Fig. 6 shows the percentages for those activities during which the students exhibited concentration and attention throughout the performance of the activity. The interest and level of attention when engaged in MTS activities is proof of the significant motivation on the part of the students, as evidenced by the concentration when doing the activities and the interest in continuing with the activities at the conclusion of the work sessions.

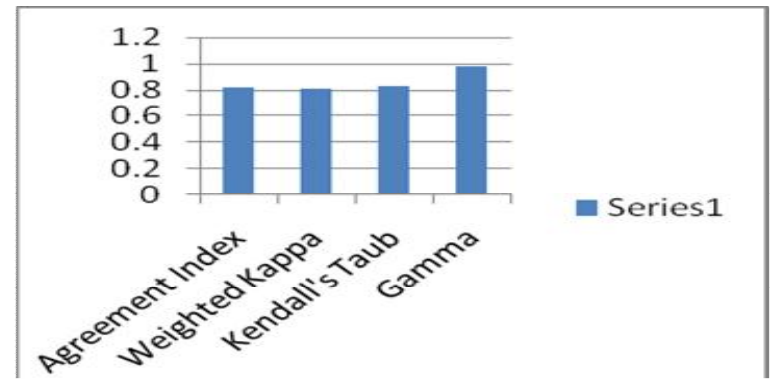

Figure 5: Validation for Fuzzy System

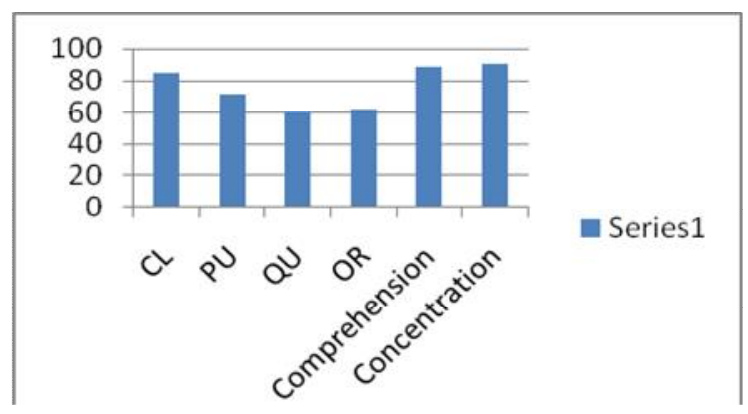

\section{CONCLUSION}

This work has presented the Fuzzy System and Multi Agent System Instructional Planner for MTS. It has been modeled to define a difficulty level in the activities to be carried out by the students. Our experience is that the teaching the Numbers and Problems are satisfactorily modeled by using the methodology Fuzzy and MAS. Motivation must also be considered when working with children. The creation of attractive interfaces, the use of multimedia resources, the fact that the activities are shown as a game, and the use of pedagogical animated agent for social interaction within the system are crucial. In future MTS can be extended using Natural Language Processing.

\section{REFERENCES}

[1] Arthur C.Graessar, Shullan LU, George Tanner Jackson. (2004) 'AutoTutor: A Tutor with Dialogue in Natural Language', Behaviour Research Methods , Instruments \& computers, pp. 180-192.

[2] Julie Main, Tharam Dillon and Simon Shiu "A Tutorial on Case-Based Reasoning "Soft Computing in Case Based Reasoning, (Eds.) Sankar K Pal, Tharam Dillon and Daniel Yeung, Springer-Verlag (London) Ltd, 2001, pp.1-28.

[3] Joice Barbosa Mendes, Alexandre Ramos "A CaseBased Reasoning Framework for Knowledge Structuring Structuring the domain module in intelligent tutoring systems." IEEE 2012.

[4] K.J.Satao et al. (2010) 'Develop English Tutorial System using NLP With Text Mining', International Journal of Engineering Science and Technology Vol.2, pp. 7149-7154.

[5] Kenji Hanakata, Guido Lorch. (2003), 'An experimental intelligent tutorial system viewed as a hyper-document', IEEE Educational Technology, pp. 7-12.

[6] Meeta Dewangan, K J Satao. (2011) 'Design and Implementation of English Language Tutorial System with Intelligent Feedback System', International Journal of Scientific \& Engineering Research Vol. 2, pp. 1-4.

[7] Michael B. Twidale. (1996) 'Knowledge Acquisition for Intelligent Tutoring Systems', Computing Department, University of Lancaster, pp. 1-8.

[8] Simon Kendel, Malcolm Creen, " An Introduction to Knowledge Engineering”, Springer International Edition.

Figure 6: Percentage of Right Answers to activities 